\title{
Los conceptos y las cosas. Evolución y alcance de la teoría vitalista del concepto
}

\author{
Axel Cherniavsky \\ Universidad de Buenos Aires
}

\section{Resumen}

Forma eterna e inmutable, representación del entendimiento, síntesis del ser y de la esencia, el concepto parece recibir una definición distinta según la filosofía que lo considere. No obstante, un esfuerzo común parece aunar las filosofías de Spinoza, Bergson y Deleuze, que de distintas maneras se proclaman vitalistas. Consiste justamente en insuflarle al concepto el máximo de vida, una plena realidad. Se determinarán en cada caso cuáles son las características de esta vitalidad, qué es lo que al concepto le confiere plena realidad, para al final evaluar las ventajas y los riesgos del nuevo concepto de concepto.

Palabras clave: concepto, representación, vitalidad, singularidad, consistencia.

\section{Abstract}

Eternal and unchangeable Form, representation of the intellect, synthesis of being and essence, the concept seems to admit a different definition in each philosophy. Nevertheless, the systems of Spinoza, Bergson and Deleuze -all vitalists but in different ways-- seem to converge on one single common effort. It consists precisely in infusing the concept with the maximum of vitality, a full reality. We will determine in each case which are the notes that 
compose this vitality, what confers the concept an absolute reality, so that we can evaluate the advantages and risks of the new concept of concept.

Keywords: concept, representation, vitality, singularity, consistency.

卫19 al vez la filosofía comience con la sospecha — tal vez no sea sino la sospecha- de que la materia observable no agota la totalidad de lo real. No pensamos en la materia no observada, en la que está en el cuarto de al lado o la que habita pasados inmemoriales y futuros lejanos. Pensamos en lo inmaterial e inobservable, Espíritu, Idea o Acontecimiento. La filosofía consistiría en la sospecha de que el amor, por ejemplo, no es pura química entre cuerpos o que los sucesivos estados de la materia no revelan el secreto del tiempo. Ulteriormente, ni siquiera el cuerpo agotaría la esencia de la corporalidad: ¿quién no se ha duchado estando limpio, por sentir sucia el alma? La filosofía aspiraría a constituirse como la especulación más rigurosa posible de aquello que por definición no puede ser observado. Aspiraría a la precisión de los métodos de la ciencia, pero su objeto la acercaría a los métodos de la religión, la poesía y la magia. Ciencia de lo suprasensible, quizá su objeto siempre sea el espíritu, en alguna de sus formas.

Incluso los materialismos, los empirismos y los realismos pueden comprenderse como un extremo de la filosofía definida como ciencia de lo suprasensible, como la otra cara de la misma moneda, como una negación de sí misma que no puede sino confirmar la afirmación. De hecho el empirismo, desde Hume hasta Wittgenstein, siempre tuvo como vocación el marcar los límites de la filosofía: sobre lo que puede y sobre lo que no puede hablar. En el otro extremo, el espiritualismo desborda estos límites, pero encuentra el problema de cómo distinguirse de una especulación no racional, de un discurso no crítico de lo inmaterial. 
Las filosofías de Bergson, de Spinoza, de Deleuze, se ubican en la convergencia de estas dos vertientes. Afirmando la plena realidad de algo que difiere por naturaleza de lo material y observable, realizan el mayor esfuerzo por lograr de ello una descripción detallada y completa. "Verdadero empirismo", llama Bergson a esta suerte de empirismo generalizado (2003: 196); " "empirismo trascendental", lo bautiza Deleuze (1968: 86). No es casual que los tres se reclamen monistas: pretenden salvar una brecha entre dos ámbitos que extraería de uno la realidad que le daría al otro. La indivisibilidad del ser tendría la ventaja de contagiarle al espíritu la innegable realidad que se le confiere al cuerpo. La unidad elemental de toda filosofía, el concepto, sería la herramienta y el signo de esta operación.

¿Qué es un concepto filosófico? El concepto es a la filosofía lo que la nota a la música y el número a la aritmética. Su unidad elemental, su átomo. Más difícil es la pregunta por su estatus ontológico: ¡es una idea y, como tal, goza de una realidad estrictamente mental que corre el riesgo de evaporarse si ve la luz del día, como las estrellas y los vampiros? ¿O bien, como creía Platón, es lo más real de todo lo real, pues es eterno e inmutable? ¿Podría, por último, sobrevivir más allá de las fronteras de la mente individual sin ser por ello ni un cuerpo ni una pieza de museo, eterna e inmutable?

Responderemos a estas preguntas trazando una breve historia del concepto vitalista a partir de algunos de sus mayores exponentes: Spinoza, Bergson y Deleuze. Por supuesto, será imprescindible recurrir a los interlocutores más concernidos de la tradición, como Platón, Aristóteles o Descartes, para determinar la especificidad del concepto que el vitalismo fue labrando con el correr de los siglos. Creemos que, de forma paulatina, éste ha ido ganando vitalidad, singularidad, consistencia, hasta abrirse paso entre los habitantes del mundo. El final de este recorrido no está exento de problemas, pero

${ }^{1}$ Salvo indicación contraria, todas las traducciones del francés son nuestras. 
al menos los problemas que el concepto enfrenta en la actualidad, son los problemas de la vida.

¿Qué es una idea para Spinoza? Comencemos por una serie de distinciones, algunas extrínsecas, otras intrínsecas, que nos depositarán sobre la vía para determinar el estatus ontológico del concepto spinozista. La primera de ellas es una distinción muy general y extrínseca, entre lo que es y no es idea, que permite comprenderla en su totalidad. Ante todo, la idea no es un cuerpo. Eso que la idea no puede ni debe ser, eso de lo que la idea se diferencia radicalmente, por naturaleza, es el cuerpo. Dos son los atributos que el hombre conoce, y visto que su intelecto es finito, no puede conocer más: el pensamiento y la extensión. Dos, por consiguiente, son los modos finitos que puede conocer: el modo que pertenece al atributo pensante, la idea, y el modo que pertenece al atributo extenso, el cuerpo; la cosa pensante y la cosa extensa. Ahora bien, gracias al paralelismo, el pensamiento y la extensión son una y la misma cosa, la sustancia, expresada de dos maneras distintas, desde dos perspectivas distintas. Y lo que vale para la sustancia vale también para los modos finitos: una idea y un cuerpo son una y la misma cosa expresada de dos maneras distintas. Las expresiones son radicalmente distintas, pero lo expresado es ontológicamente uno. Distintos por naturaleza pero inseparablemente ligados: tales son las ideas y los cuerpos.

Cuando un cuerpo impacta en otro cuerpo, este impacto produce una afección, a nivel material, y una idea de esa afección, a nivel mental. Llegamos así a una segunda distinción, intrínseca ahora, una distinción entre las ideas que tenemos. Estas pueden ser de tres tipos según Spinoza, quien divide el conocimiento en tres géneros. $\mathrm{Al}$ primer género de conocimiento lo componen las ideas de las afecciones y concierne a la imaginación. Este tipo de ideas no me informa 
sobre la naturaleza del cuerpo afectante ni sobre la naturaleza del cuerpo afectado, sino pura y exclusivamente sobre la afección. No por este género sabemos que el hielo es hielo y que mi cuerpo es como mi cuerpo, sino simplemente que el hielo es frío o que, mejor dicho, siento algo frío. El segundo género de conocimiento remite al intelecto o entendimiento, cuyos productos son las nociones comunes. No deben confundirse con las ideas universales o abstractas, sino que son ideas que permiten conocer las propiedades comunes de las cosas. Por último, la facultad del tercer género de conocimiento es la intuición o ciencia intuitiva y sus productos son las ideas de las esencias de las cosas singulares. Como las ideas del primer género de conocimiento, las del tercero apuntan a las cosas singulares; pero a diferencia de ellas, éstas sí permiten conocer la naturaleza del cuerpo afectante o del cuerpo afectado por ejemplo. Son tres las ideas del tercer género: la idea de la esencia de Dios, la idea de la esencia de mí mismo y la idea de las esencias de las cosas. Todas estas ideas, las de los tres géneros, son modos representativos: representan un determinado estado de cosas, ya sean cuerpos, o ideas, y en este caso, tenemos ideas de ideas. Pero hay otros modos del atributo Pensamiento que no son representativos. Debemos recurrir a una tercera distinción, extrínseca de nuevo, entre lo que las ideas son y lo que no son, pero ya dentro del atributo pensante.

Por un lado, el alma tiene ideas propiamente dichas, que son modos representativos, a las que corresponde un objeto. Pero por otro, contiene afectos o sentimientos que, en estricto sentido, no representan nada. Por supuesto, un amor es inseparable de un objeto amado, como un temor es inseparable de un objeto temido (Spinoza, 1999, II, axioma 3). Pero no por ello debemos confundir al amor con su objeto o con la idea de ese objeto. Spinoza define al amor como "una felicidad que acompańa la idea de una causa exterior" (III, proposición 13, escolio), lo cual permite distinguir al amor, que es una 
felicidad, de la idea de la causa exterior, que es la idea del objeto y de la causa exterior, que es el objeto. El afecto propiamente dicho es un sentimiento, una variación de la potencia de actuar y el amor en particular, una felicidad, es decir un aumento de la potencia. Deben diferenciarse en este sentido las ideas, modos representativos del pensamiento, de los afectos o sentimientos, que no representan nada sino que son aumentos o disminuciones de la potencia. Ahora bien, en la medida en que todo es potencia en Spinoza, en la medida en que la sustancia es la potencia universal, cada cosa como parte de esa sustancia, es una expresión de su potencia. Cada modo finito es un grado o intervalo de potencia y, en este sentido, también las ideas lo son. Así, no desde esta perspectiva las ideas se distinguen de los afectos o sentimientos, sino exclusivamente desde el punto de vista de la representación. Por este mismo motivo, Spinoza rechaza la distinción cartesiana entre las ideas y los juicios. Antes de proceder a una distinción intrínseca entre las ideas (innatas, adventicias o facticias) (Descartes, 1979: 101), Descartes distingue la idea a la cual le "conviene propiamente el nombre de idea", de la volición y del juicio (99). No es el caso en Spinoza, para quien la idea es ella misma la afirmación o la negación de su objeto. Gracias a una teoría del conatus generalizada, Spinoza habilita a una experiencia de la idea, a un sentir la idea.

Hemos considerado las ideas desde un punto de vista ontológico y desde un punto de vista gnoseológico. Según el primero, las ideas se distinguen de los cuerpos y son un modo finito del atributo pensante. Como tales, son una expresión parcial de la universal potencia de pensar. A partir del segundo, las ideas se distinguen de los sentimientos en tanto representan un objeto, afección, propiedad común o esencia, según pertenezcan al primer, al segundo o al tercer género de conocimiento. Pero no se distinguen de ellos ni de los juicios en tanto ellas mismas son la expresión de una potencia. Como tales, son la afirmación o la negación de su objeto. 
Tal vez ahora, gracias a estas distinciones, estemos en condiciones de evaluar el estatus ontológico de la idea en Spinoza. Creemos que, en primer lugar, con Spinoza la idea se vuelve más consistente. Es cierto, la distinción escolástica entre la realidad formal y la realidad objetiva que Descartes retoma (105) ya dirigía nuestra atención sobre el ser de la idea, sobre la idea como ser, y no sobre su carácter representativo o reflexivo. Por otra parte, ya Platón hacía de las Ideas o Formas el ser más elevado, por sobre las apariencias, los cuerpos y demás cosas del mundo sensible sujeto al devenir. En este sentido, el tenor de realidad de la Idea platónica parece insuperable. Pero con Spinoza la idea se hace cuerpo. En efecto, el llamado "paralelismo" es al respecto un arma de doble filo: distingue lo inseparable tanto como acerca lo inconfundible. Ideas y cuerpos son radicalmente distintos, existe entre ellos una diferencia que no es de grado, sino de naturaleza. Pero, al mismo tiempo, uno y otro son la expresión de lo mismo, tanto a nivel sustancial como a nivel modal. Tal es así, que el vocabulario que emplea Spinoza en un caso y en otro es el mismo: cuerpos e ideas, ambos son "modos finitos", ambos "determinan" a sus pares (III, proposición 2). ¿Hay una ganancia de realidad o de ser por parte de la idea? No es del todo cierto: desde una perspectiva platónica, en donde nada es más que la Idea, en donde lo fugaz, lo evanescente es el cuerpo, la afirmación no tiene sentido. ¿Hay en efecto una ganancia de corporalidad? Se nos perdonará la expresión, que es sin duda metafórica, y que lo último que pretende es confundir lo distinto; pero decimos que la idea se hace cuerpo, no para quitarle especificidad a la idea en relación al cuerpo sino, al contrario, para devolvérsela en función de la tradición. Quizá sea más conveniente afirmar que la idea, en Spinoza, experimenta una ganancia de consistencia o tangibilidad.

En segundo lugar, la idea spinozista es más móvil o vital. Para Descartes las ideas eran copias de las cosas, "cuadros" (1979: 109). 
Spinoza critica de forma explícita esta concepción: "las ideas no son como pinturas mudas sobre un cuadro" (II, proposición 49). No lo hace, sin embargo, por el hecho de que la idea tenga un carácter representativo; en este sentido, Spinoza participa plenamente del aire de su tiempo. El Pensamiento sigue siendo una suerte de espejo universal que lo refleja todo y que se refleja incluso a sí mismo, según lo que Martial Guéroult denomina como el "paralelismo intracogitativo" (Guéroult, 1968: 64-72). El acento, en la crítica de Spinoza, no ha de ponerse en el sustantivo "pinturas", sino en el adjetivo "mudas". Y a lo que apunta es a la rigidez, a la estabilidad de la idea cartesiana, a su silencio. Spinoza, en cambio, quiere una idea locuaz, una idea viva. Es por eso que anuda la idea a la volición (II, proposición 49, corolario, demostración) y el intelecto a la voluntad (II, proposición 49, corolario). Si Descartes distingue a las ideas de los juicios, Spinoza afirma la identidad de la idea con la volición, entendiendo por volición lo que el primero entiende por juicio: afirmaciones o negaciones. Las ideas spinozistas no son pinturas mudas sino retratos elocuentes, llevan consigo la afirmación o negación de su objeto.

La diferencia con el primer Platón es evidente si recordamos que las Ideas, en su caso, son y deben ser inmutables (Fedón, 78d; Cratilo, 439c-e). Al mismo tiempo, no podemos pasar por alto las correcciones que Platón introduce respecto de su propia teoría en el Sofista. Curiosamente, afirma allí

que existe realmente todo aquello que posee una cierta potencia, ya sea de actuar sobre cualquier cosa natural, ya sea de padecer, aunque sea en grado mínimo y a causa de algo infinitamente débil, incluso si esto ocurre una sola vez. Sostengo entonces esta fórmula para definir las cosas que son: no son otra cosa que potencia (Sofista, 247d-e) $)^{2}$.

2 Se trata de una traducción privada al español de Néstor Cordero. Traduce su propia traducción del texto griego al francés, en (Platón, 1993: 153). 
Las cosas que son, son las ideas. He aquí entonces que las Formas, durante tanto tiempo eternas e inmutables, se vuelven potencia, capacidad de afectar y ser afectadas. Ahora bien, ¿de qué tipo de movimiento se trata? En realidad, el término movimiento ya les queda demasiado grande a las Ideas, pues se trata más bien de un movimiento sin traslación, de un vibrar, de un latir. Platón, en este sentido, no puede ser más que un antecedente de Spinoza, en donde las ideas se mueven de verdad, afectandose unas a otras, formando cadenas causales que constituyen un verdadero intercambio de ideas, comercio de ideas. ¿Qué es Ética III sino el mapa del alma, con sus avenidas principales, una verdadera física mental? Por otra parte, lo que en Platón es una corrección ulterior, en Spinoza es la base de su teoría del conocimiento: un cierto dinamismo en el primero, un verdadero mecanicismo en el otro. Diremos por lo tanto que, en relación a Platón o a Descartes, la idea spinozista se muestra especialmente móvil y vivaz.

Por último, dirijamos nuestra atención a la posición de la idea y a su relación con el alma humana en particular. En Platón, por más dinámicas que sean, las ideas siempre estarán en lo alto, como dice Bergson (2003b: 38), poblando el Cielo. Cayeron con tal fuerza que, en Descartes, se metieron dentro de la cabeza de los hombres y perdieron su movilidad. Las ideas, ahora, se tienen: los límites de su territorio son la corteza cerebral. En la filosofía de Spinoza, no sólo recuperan su movilidad, sino que se vuelven tan ágiles que a veces escapan a los hombres. En efecto, en Spinoza, las ideas que no tenemos gozan del mismo tenor de realidad, de la misma consistencia que las que tenemos. Son las ideas inadecuadas, que están en Dios como no están en nosotros, y las ideas que no conocemos, que son en Dios, en el pensamiento, sin ser en nosotros. Diremos que las ideas bajaron a la tierra sin humanizarse por ello. No perdieron su divinidad, porque el pensamiento, en Spinoza, es Dios, es decir la sustancia. Pero gana- 
ron un carácter terrenal, porque si el cielo en Platón es trascendente, en Spinoza, es inmanente. Quizá lo mejor sea decir que se naturalizaron: bajaron de un cielo trascendente y no se limitan a las fronteras del alma humana, sino que habitan el atributo intelectual de Dios, es decir la naturaleza. Transitan por el universo del pensamiento, que es tan consistente como el de la extensión, aunque inmaterial. A veces entran en el espíritu de un hombre, como a veces un pájaro se mete dentro de una casa por la ventana, a veces se escapan, como el perro a su hogar, a veces viven libres y errantes, en la infinitud del pensamiento, como animales salvajes.

En el sistema de la sustancia, las ideas han ganado consistencia, pues su orden y conexión son los mismos que los del cuerpo; han ganado movilidad o vitalidad, pues tienen la capacidad de afectar y ser afectadas por otras ideas, en cadenas causales que se prolongan al infinito; y han ganado terrenalidad o inmanencia, pues su caída desde lo alto no implica un aprisionamiento en las profundidades de la mente humana. Sin duda, por todos estos motivos, afirma Bergson que hay en Spinoza "impulsos de intuición que resquebrajan el sistema” (Bergson, 2003c: 346).

¿Qué es la intuición en Bergson? La intuición debe ser ante todo distinguida de la intuición y de la intuición. Es que Bergson emplea el mismo término para tres elementos distintos: un método, una facultad y el producto de esa facultad.

La intuición es el método de la filosofía, y retomando un léxico spinozista, Bergson dice que consiste en percibir sub specie durationis (2003: 176). Intuir es pensar "en durée" (30). Por un lado, la intuición como método de la filosofía se opone al análisis como método de la ciencia (181). La ciencia busca rivalizar con la naturaleza, dominarla, mientras que la filosofía pretende simpatizar con ella, comprenderla. 
Por otro lado, la intuición debe asimismo diferenciarse de la percepción artística, que si bien dilata nuestra percepción, mostrándonos lo que con frecuencia no vemos al mirar, lo hace sólo en superficie. La intuición agrega una dimensión a la percepción artística, el tiempo, y la amplía en profundidad (175). La intuición como método es la comprensión temporal del tiempo, la percepción cambiante del cambio.

Como facultad, la intuición consiste en la captación inmediata de lo singular y lo moviente, de lo esencial a todas las cosas y a cada cosa. Desde este punto de vista, se opone a la inteligencia, que divide y homogeniza un objeto que debe darse simultáneamente. El objeto de la inteligencia es el espacio, la materia. El de la intuición no es un objeto, sino un proceso, el tiempo o espíritu.

Finalmente, la intuición es el producto de la intuición como facultad. Desde esta perspectiva se opone al concepto, como producto de la inteligencia e instrumento del análisis. El concepto, con sus límites bien precisos, encapsula lo real, lo estabiliza, lo identifica, lo define. La intuición, al contrario, intenta dar cuenta de su carácter heterogéneo y sucesivo, continuo y fluctuante.

Bergson dudó mucho antes de bautizar intuición a la intuición (25), tantas eran las capas de sedimento con las que la historia de la filosofía había recubierto el término. Distingamos entonces la intuición bergsoniana de las intuiciones de la tradición. La intuición de Bergson no es como la de Descartes, una intuición intelectual. Dos actos cognoscitivos ofrecen las reguale cartesianas para acceder a las cosas sin temor a equivocarse: la deducción y la intuición. Pero ambos son actos del entendimiento. "Por intuición, entiendo [...] el concepto que la inteligencia pura y atenta forma con tanta facilidad y distinción que no queda absolutamente ninguna duda sobre lo que comprendemos [...]" (Descartes, 1996: 14) La definición de la intuición, pero también los ejemplos que Descartes ofrece, por lo general tomados de la geometría y la aritmética, permiten pensar la 
intuición como una deducción veloz. En efecto, ella no carece de una cierta discursividad. Intuición y deducción son la bifurcación que tiene como raíz común al entendimiento. La diferencia entre una y otra es gradual. La intuición de Bergson, en cambio, se distingue por naturaleza y no por grados del razonamiento intelectual. La inteligencia divide, homogeneiza y estabiliza. Es en esencia práctica. La intuición, esencialmente especulativa, capta lo indivisible y sucesivo. Inteligencia e intuición no son dos operaciones de una misma facultad, sino dos facultades distintas. Bergson, como Descartes, cree en la posibilidad de captar la cosa tal cual es; pero el segundo gracias a una operación intelectual, el primero gracias a una facultad supraintelectual.

En segundo lugar, la intuición bergsoniana debe distinguirse de la platónica (episteme o noesis): si bien ambas son una captación inmediata, lo que capta una es eterno e inmutable, idéntico a sí, y lo que capta la otra es temporal y cambiante, eternamente distinto de sí. En relación a Kant, debemos decir que la intuición no capta el fenómeno, sino lo que para Kant es la cosa en sí. En efecto, Bergson, como Husserl, considera el criticismo como un desafío, e intentan refundar un conocimiento absoluto. Por momentos, al leerlos, pareciera que estamos ante filosofías pre-críticas. "A las cosas mismas", declara la consigna fenomenológica, que es igualmente válida para Bergson, cuya intuición es una aprehensión de la esencia de cada cosa. Debemos remitirnos más al Kant de la Crítica del juicio que al de la Crítica de la razón pura y, en este sentido, no es curioso que Bergson recurra al arte para señalar un ámbito en donde las cosas se exhiben tal cual son y no mediadas por símbolos como en la ciencia. Finalmente, la intuición bergsoniana ha de distinguirse de la spinozista, en tanto que la segunda es una intuición de las esencias de las cosas singulares y la primera, para ponerlo en los mismos términos, una intuición de las esencias singulares de las cosas. En efecto, como 
bien explica Guéroult, nada indica que, en la Ética, la ciencia intuitiva nos ofrezca las esencias de cada cosa, en vez de la esencia singular de las cosas (Guéroult, 1968: 458-463). No es el caso en Bergson, en donde la intuición se amolda a cada objeto, se mimetiza con cada proceso.

El concepto es el producto de la inteligencia y la herramienta de la ciencia; la intuición, la producción de la intuición como facultad y el instrumento de la metafísica. La ciencia analiza la materia y el espacio; la metafísica simpatiza con el tiempo y el espíritu. El análisis divide, unifica y pone en simultáneo lo que la intuición descubre como indivisible, plural y sucesivo. La ciencia, dice Bergson, se aproximará indefinidamente a lo real, multiplicando perspectivas y variando puntos de vista: nos ofrecerá siempre un conocimiento relativo. La filosofía, en cambio, nos deposita en el interior de lo real, proveyéndonos de un saber absoluto. Se puede traducir una novela en cientos de lenguas, ninguna traducción nos impactará como el original. Podremos sacar miles de fotografías de una ciudad, ejemplifica Bergson, pero nunca será como pasear por ella (2003: 179). Con cuadros y pinturas comparaba Descartes las ideas, de fotografías distingue Bergson a la intuición. Es esta diferencia la que nos permite evaluar el primer aporte de Bergson para la construcción de un concepto vitalista.

La idea, tanto en Spinoza como en Descartes, al igual que lo que Bergson entiende por concepto, es una representación de lo real, una copia de su objeto. Poco importa la psicología implicada, si debe haber un estímulo sensible o no, si el cuerpo le transmite la imagen al alma o constituye el alma un circuito "paralelo". En todos los casos, la idea aparece como un reflejo de lo real, copia o duplicación. La particularidad de la intuición bergsoniana es que no representa, sino que presenta lo real, es una mímica y no una imitación. En segundo lugar, con Bergson, la idea parece haberse afinado, agudizado, pues ha 
perdido todo carácter unificador o totalizador. Ya Spinoza distinguía sus nociones comunes de las ideas abstractas o generales, pero éstas bien eran "comunes" a todas las cosas. Nada ha quedado de esto en Bergson, nada del concepto kantiano que subsume las intuiciones: a cada cosa una intuición. E incluso la intuición es lo que nos permite decir que lo que creíamos que era una cosa, en realidad, eran muchas, fugaces, únicas, irreversibles. Por otra parte, ese carácter corpóreo o material de la idea spinozista, su consistencia, se ve conservado en la filosofía de Bergson. El "empirismo verdadero" es un esfuerzo por darle a la metafísica la precisión de la ciencia y al espíritu, el color y la textura de la materia. Hay un dualismo tanto en Spinoza como en Bergson, de atributos en el primero, de tendencias en el segundo, pero en ambos casos es provisorio: el primero se resuelve en la unidad de la sustancia, el segundo en la simplicidad del élan (Jankélévitch, 1959: 174). En cierto sentido, Bergson dinamiza un sistema que ya era dinámico, aceita un mecanicismo, lo "organiza”. Al reemplazar las causas por procesos, hace del mecanismo universal un organismo universal: viste esa Naturaleza de la cual Spinoza había dado sólo el esqueleto. El resultado de ambos monismos es tanto la espiritualización de la materia como, lo que aquí nos importa, la materialización del espíritu. Por eso decimos que la consistencia de las ideas se encuentra totalmente conservada. Pero, finalmente, ese carácter que denominamos terrenal o inmanente de las ideas, por el cual significamos que no están ni el cielo ni en la mente de los hombres exclusivamente, se ha perdido, pues la intuición de Bergson es el producto de una facultad que aparece sólo con el advenimiento del hombre (Bergson, 2003c, cap. III).

Ganancia en movilidad o singularidad; conservación de consistencia y pérdida de terrenalidad: tal es el pasaje de la idea spinozista a la intuición de Bergson. La originalidad de ésta reposa en su carácter no representativo. Es un heredero de ambos filósofos, Deleuze, quien 
va a agrupar todos estos caracteres en su concepto de concepto, y a aportar por otra parte innovaciones propias.

En cierta medida, Diferencia y repetición puede leerse como una movilización deleuziana de Bergson contra Hegel. En efecto, Deleuze intenta pensar un concepto de la diferencia que no requiera de un pasaje por las nociones de negación, oposición o contradicción. Hay una clase de diferencia que captamos con facilidad, y que constituye un concepto indispensable para todas las operaciones prácticas de nuestra vida cotidiana. Es el que se expresa en una proposición del tipo " $a$ es diferente de $b$ ", una casa es diferente de un auto, un golpe es diferente de una caricia; esta idea de diferencia implica de alguna manera la idea de una negación y la idea de una exterioridad: un auto es diferente de una casa en tanto no es una casa; una cosa es la casa, otra el auto, lo uno no es igual o idéntico a lo otro — admitamos por un momento que una identidad de este tipo sería posible-, por lo tanto, son diferentes. Bergson ha dedicado buena parte de sus esfuerzos filosóficos a pensar otra clase de diferencia, una diferencia que no requiera ni de la negación ni de la exterioridad, una diferencia interior a una cosa que en cierta medida parece ser la misma. Quizá, el caso más ilustrativo sea el del envejecimiento. Cuando una persona envejece decimos que ha cambiado, que es diferente. No nos referimos, al menos en este caso a que es otra persona: es la misma pero diferente. Tal es la durée y todos los fenómenos que de ella dependen: cuantitativamente una y cualitativamente diferente. He aquí una nueva clase de diferencia, una diferencia que no se da entre $a$ y $b$ sino en el interior de $a$, una diferencia de $a$ respecto de sí misma. A la relación de oposición o negación dialéctica, Deleuze va a oponer entonces, de manera no dialéctica, un concepto diferente de la diferencia, una diferencia intrínseca y positiva. 
Ahora bien, al buscar un nuevo concepto de la diferencia, un concepto que no la subordine a la negación, a la oposición, Deleuze va a dar al mismo tiempo con un nuevo concepto de concepto. Para crear un nuevo concepto de la diferencia, hay que crear un concepto diferente de concepto, esto es, un concepto que pueda él mismo llevar la diferencia, cargar con la diferencia. Eso que es diferente de sí, también es el concepto mismo. Así, mientras Deleuze descarta las concepciones de la diferencia que pueda ofrecer la historia de la filosofía, como Platón con las Ideas o Aristóteles con los géneros y especies, porque siempre subordinan la diferencia a la identidad o mismidad, inventa un nuevo concepto, distinto de la Idea, del género, de la especie, pues lejos de ser idéntico a sí, lleva en sí mismo la diferencia. Es por este motivo que creemos que Deleuze conserva una propiedad de la intuición bergsoniana, la singularidad. Las divergencias en el léxico no deben despistarnos. Lo que muchas veces Deleuze llama idea en Diferencia y repetición, es lo que llamará concepto en ¿Qué es la filosofía?, y no corresponde al concepto bergsoniano, sino al contrario, a la intuición. Sin embargo, en sus declaraciones explícitas, Deleuze no atribuye este descubrimiento a Bergson, sino a Leibniz y a los teóricos del concetto: "el concepto no es un simple ser lógico, sino un ser metafísico; no es una generalidad o una universalidad, sino un individuo" (Deleuze, 1988: 56).

La cita anterior, proveniente de El pliegue, muestra en segundo lugar que Deleuze permanece bergsoniano en relación a la representatividad o no representatividad del concepto. "No es un ser lógico, sino un ser metafísico". Por un lado, esto significa que es un individuo, una singularidad, un particular, pero también desvía nuestra atención de la pretendida capacidad representativa del concepto, de orden lógico, a su plena existencia como ser en sí. Es que, en efecto, el concepto deleuziano no refiere a nada más que a sí mismo, no representa nada sino que se presenta él mismo: "el concepto se define por 
su consistencia, endo-consistencia y exo-consistencia, pero no tiene referencia: es autoreferencial, se plantea a sí mismo y plantea su objeto, al mismo tiempo que es creado" (Deleuze y Guattari, 1991: 27) ${ }^{3}$. La endo-consistencia remite a la conexión interna de los elementos que componen al concepto; la exo-consistencia, a la conexión externa del concepto con otros conceptos. Poco importan estas precisiones aquí, pues con la noción general de consistencia Deleuze quiere redirigir la atención del objeto del concepto al concepto mismo, de su pretendida representatividad a su presencia. El concepto deleuziano entonces, como la intuición bergsoniana pero a diferencia de la idea spinozista, no representa a nada más que a sí mismo. O mejor, ni siquiera consigo mismo está en una relación de representación, sino de plena afirmación.

Ahora bien, en tercer lugar, la noción deleuziana de concepto recupera una característica de la idea spinozista que la intuición bergsoniana había perdido: su terrenalidad o inmanencia. Vimos que la intuición bergsoniana, como la idea cartesiana, estaba, por así decirlo, encerrada en la cabeza de los hombres. Sin devolvernos a la trascendencia de la Idea platónica, Deleuze vuelve a conferirle al concepto una terrenalidad no humana, la capacidad de transitar por el mundo sin entrar necesariamente en conexión con la cabeza de los hombres. En efecto, si bien Deleuze insiste en que la filosofía es la actividad que crea conceptos, se cuida de no hacer depender esta creación de un autor entendido como un sujeto, res cogitans, en total dominio de sí que un buen día se determina voluntariamente a crear conceptos. Es allí donde interviene la teoría del personaje conceptual. El personaje conceptual es una suerte de heterónimo del filósofo por medio del cual éste crea sus conceptos. Remite a la cuarta

${ }^{3}$ El verbo que traducimos por plantear es poser. En francés, poser significa literalmente 'apoyar', lo cual en este caso le da un sentido mucho más material al concepto, que el verbo plantear no logra traducir. 
persona del singular de Blanchot y funciona como el on impersonal del que dispone la lengua francesa. Del concepto deberemos decir que crea o se crea de la misma manera que decimos llueve (Deleuze y Guattari, 1991: 62-63). Ahora bien, la creación del concepto podría estar en manos de un sujeto desposeído que no por ello sería menos un sujeto. El concepto bajaría a tierra por una especie de pararrayos, pero ese pararrayos seguiría siendo un hombre. Por eso debemos remitirnos a lo que dice Deleuze de las creaciones artísticas en general y luego trasladarlo al arte filosófico. Las producciones del arte son los afectos y perceptos. Deleuze utiliza estas palabras para distinguir lo que quiere decir de las afecciones y percepciones. "Los perceptos ya no son percepciones, son independientes de un estado de los que los experimentan; los afectos ya no son sentimientos o afecciones, desbordan la fuerza de esos que pasan por ellos. Las sensaciones, perceptos y afectos, son seres que valen por sí mismos y exceden toda vivencia." (Deleuze y Guattari, 1991: 154). ${ }^{4}$ De hecho, el objetivo del arte es extraer el percepto a la percepción y el afecto a la afección. $\mathrm{El}$ artista debe lograr que una tristeza flote en el aire, entre la obra, el artista y el espectador, independiente de los tres, capaz de atravesar las fronteras del triángulo. Quizá por eso la música, tan movediza y tan invisible, tan volátil y tan incorpórea a los ojos de los hombres, sea en tantas ocasiones el caso límite de las reflexiones de Deleuze sobre el arte. De todos modos, es esto mismo lo que debemos pensar del concepto. Terreno e inhumano, encuentra a los hombres pero también todo lo demás que los hombres encuentran, y tal vez incluso cosas que no.

Por último, ahora sí en una línea que une los nombres de Spinoza, Bergson y Deleuze, este último le confiere al concepto una plena realidad que más que nunca querremos llamar materialidad o corporalidad. Al igual que sus predecesores no duda en admitir un ${ }^{4}$ El subrayado es del original. 
ámbito distinto del de la materia y los cuerpos que llamará virtual. En efecto, lo virtual debe ser alineado con el atributo pensamiento de Spinoza y con el espíritu de Bergson. Pero al igual que sus predecesores, Deleuze estará en lucha constante con un dualismo radical e intentará por todos los medios fundir sin confundir este ámbito con el de los cuerpos, el de la materia, que Deleuze llama actual. ${ }^{5}$ Por eso, utilizando la frase de Proust dirá de lo virtual que es "real sin ser actual" y, comentando a Spinoza, podrá afirmar "cuanto más matemático, más concreto". ${ }^{6}$ Ahora bien, desde el punto de vista lexical existe una diferencia importante entre Deleuze y Bergson, que en realidad es indicio de una diferencia más importante, de un movimiento de radicalización. A la hora de escribir o de hablar, Bergson se encuentra con un problema discursivo que podría expresarse así: ¿cómo expresar el tiempo o el espíritu que es cambiante y sucesivo con una lengua que es homogénea y simultánea? Bergson entiende que esa lengua admite luego expresiones disímiles: la de la ciencia, la del sentido común, en síntesis, la del espacio, que nunca podrá expresar las verdades de la intuición, y la de los poetas, la de los novelistas, la del arte, que estará mucho más cerca de lograrlo. El discurso filosófico deberá ser entonces, en alguna medida, poético para expresar los descubrimientos de la metafísica. Y es así que, tanto Bergson como sus comentaristas, constatan el poder y la utilidad de la metáfora. Por un lado Bergson va a separar y reservar algunos términos para el ámbito espiritual (heterogéneo, continuo, ligero...) y otros para el espacial (homogéneo, divisible, pesado...); y por otro, va

${ }^{5}$ Esta consideración del espíritu en términos materialistas condujo a ciertos comentadores a expresarse, no sin timidez, sin introducir las palabras que el filósofo evitó, en términos de una materialidad de lo virtual (Alliez, 1998: 49) o de una materialidad incorpórea (Foucault, 1994: 947).

${ }^{6}$ Es la clase consagrada a Spinoza en la Universidad de Vincennes, el 24 de enero de 1978. Disponible en el webdeleuze: http://www.webdeleuze.com/php/texte. php cle $=11 \&$ groupe $=$ Spinoza\&langue $=1$. 
a utilizar distintas metáforas para expresar la durée (la musical es las más célebre de todas). A diferencia de Bergson, Deleuze, cuando tenga que expresar la naturaleza del acontecimiento por ejemplo, o de algún fenómeno virtual, se referirá a él como una temperatura, una velocidad un color, una intensidad. Los términos no podrían ser más materiales: todos tienen su origen en la física o en la óptica. Y como si fuese poco, Deleuze va a insistir en que no se trata de metáforas. Sin duda, un autor y otro entienden de manera distinta la metáfora. Para Deleuze, la metáfora remite a lo imaginario, a lo irreal, y tiene en tal caso el defecto de quitarle realidad a eso que más la necesita. Este es el motivo por el que Deleuze elige el vocabulario más material posible para hablar del espíritu, más físico posible para hablar de la metafísica: para fundir sin confundir. En efecto, es la confusión del principiante el riesgo de esta expresión, pero la máxima concreción de lo espiritual, su mérito. Después de todo, "la geografía también es mental” (Deleuze y Guattari, 1991: 91). Deleuze se ubica así en un linaje que podemos llamar materialismo espiritual, y que después de todo no es más que la proyección en un plano ontológico de lo que él mismo llama empirismo trascendental. Esta pertenencia es la que nos permite afirmar que conserva y radicaliza la consistencia del concepto hasta el punto de prácticamente dotarlo de, ahora sí, una cierta corporalidad.

Resulta de la yuxtaposición de las características enumeradas, una exhaustiva descripción del concepto en el capítulo primero de ¿Qué es la filosofía? "El concepto se define por la inseparabilidad de un número finito de componentes heterogéneos recorridos por un punto en sobrevuelo absoluto, a velocidad infinita" (26). Ante todo, el concepto no es simple sino complejo, se compone por una multiplicidad de elementos, sus componentes. Un concepto puede perder componentes, ganar componentes y quizá hasta, con el correr del tiempo, reemplazar todos sus componentes. La movilidad de 
los componentes del concepto define su historia. Ahora bien, estos componentes son "heterogéneos", diferentes entre sí y, sin embargo, "inseparables". Se funden sin confundirse. Por eso, además de una historia, el concepto tiene un devenir. Muchas veces, como en el devenir-animal por ejemplo, el devenir no consiste en un proceso temporal, sino en una alianza espacial no recíproca de elementos heterogéneos. Tal es el caso con el concepto. El devenir concierne a la alianza de sus componentes, la endo-consistencia del concepto. El concepto, posee luego una exo-consistencia, que es la conexión que entabla con otros conceptos. La exo-consistencia es la estructura del sistema filosófico, una multiplicidad de conceptos. Es importante entender que no es un nexo lógico ni cronológico, sino una relación no siempre lógica que los conceptos entablan en el espacio filosófico. El concepto, en cuarto lugar, es "el punto de condensación o de acumulación de sus propios componentes" (1991: 25). Sin ser una unidad o una totalidad, sin ser pensado como un organismo, el concepto es una multiplicidad, múltiple y una, una multiplicidad cuya unidad no es más que la conexión, el "sobrevuelo a una velocidad infinita" de sus componentes. Velocidad o condensación, es de naturaleza virtual: es lo que de Deleuze entiende por velocidad infinita. Ninguna velocidad es infinita en el ámbito material: un cuerpo puede viajar rápido, muy rápido, increíblemente rápido, pero sólo el pensamiento alcanza una velocidad infinita, una velocidad que, por ser infinita, no es estrictamente una velocidad. "El concepto es un incorpóreo" (26). Para terminar, "el concepto no es discursivo" (27), no es una proposición. La proposición tiene como objeto un estado de cosas, un referente, mientras que el concepto no refiere a nada más que a sí mismo.

Spinozista y bergsoniano, Deleuze agrupa y a veces exacerba las características previas: consistencia o materialidad, inmanencia o terrenalidad, singularidad o movilidad, no representatividad. Pero si Deleuze sostiene que la filosofía es creación de conceptos, debe 
ser ante todo deleuziano y es su propio deleuzianismo lo que debe decidir cómo hacer funcionar la historia de la filosofía. Lo cual nos conduce a la determinación del aporte irreduciblemente deleuziano a la constitución de un concepto vitalista: la funcionalidad. Un libro de filosofía, para Deleuze, es una herramienta, un instrumento, una máquina; ante todo, debe funcionar (Deleuze y Guattari, 1980: 10). Una filosofía debe funcionar y, por lo tanto, sus componentes, los conceptos, deben funcionar, servir, deben ser útiles, eficaces, resistentes. Como todo instrumento, deben servir para hacer algo con algo que no es él mismo, debe ajustar algo, cortar algo, pegar algo. Por eso insiste tanto Deleuze sobre la conexión de la filosofía con los otros ámbitos, ciencia o arte, en la aptitud de la filosofía para ofrecer el concepto de una función o de una sensación (Deleuze y Guattari, 1991: 188). El concepto debe ser una cosa entre las cosas, herramienta entre las herramientas y, como tal, su naturaleza es eminentemente práctica o, más que práctica, funcional. "Podríamos decir que las otras filosofías se ocupan de las cuestiones del mundo, de todo tipo de cuestiones, mientras que esta no se ocupa estrictamente de nada: no juzga ni transforma el mundo, lo efectúa de otra manera, como universo virtual de los conceptos" (Nancy, 1998: 119). Tal es la vuelta de tuerca entre un mecanicismo como el de Spinoza y un maquinismo como el de Deleuze que nos lleva bien lejos de la vida contemplativa aristotélica y que nos hace pasar de una experiencia de la eternidad a una experiencia de lo intempestivo, de la divinidad a la tierra y por último a la mundanidad.

El monismo de Spinoza, de Bergson y de Deleuze realiza un esfuerzo por fundir en un único plano, sustancia o tendencia, el alma y el cuerpo. El concepto filosófico es arrastrado por el mismo movimiento. Recibe así un tenor de realidad, una carga ontológica que deno- 
minamos consistencia. Existían antecedentes de ello en la Idea platónica o en la realidad formal escolástica. Pero nada parecido a una velocidad, una intensidad o una textura del concepto, adjetivación propia a Deleuze pero que no hace más que exacerbar una operación que comienza con Spinoza. Por otra parte, la Idea platónica planeaba en los cielos. El concepto vitalista, satánico o adánico, ha descendido desde lo alto para transitar la superficie terrestre. Singular, único e irremplazable, ha ido conquistando los atributos que otrora le correspondían al hombre, al sujeto, al ser humano, para luego liberarse incluso de las coordenadas personológicas y subjetivas para adquirir las propias. Flujo y no reflejo, presencia y no representación, el concepto ha devenido cosa entre las cosas, útil entre los útiles, habitante del mundo y engranaje de la vida.

Resignar el carácter representativo del concepto presenta dos ventajas de orden metafilosófico. En primer lugar, el concepto se vuelve autónomo. Al dejar de asumir la responsabilidad de representar contenidos ajenos, la filosofía no debe responder más que por sí misma. Ni clarificación de un enigmático lenguaje artístico, ni refinamiento de reclamos de clase, la filosofía podría atender sus propios problemas.

En efecto, este movimiento va de la mano con la resignificación de la figura del intelectual. Tanto para Deleuze como para Foucault (Deleuze, 2002: 290), el intelectual contemporáneo ya no se constituye, como en la época de Sartre, como el portavoz de una clase incapaz de expresar sus propios reclamos; sólo lleva la misma lucha en otro terreno, pues la fuerza que se enfrenta presenta muchas formas y se despliega en distintos terrenos. Al mismo tiempo, en segundo lugar, el resto de las actividades ganan en dignidad cuando el concepto filosófico ya no pretende estar en el lugar de otra cosa. Ciencia madre, árbol de la sabiduría u ontología fundamental, de Aristóteles a Heidegger la filosofía se autoasignó el privilegio de fundamentar 
las ciencias o de explicar al arte, cuando las primeras no necesitan fundamentación y el segundo no necesita explicación. Al no hablar el concepto más que de sí mismo, no es sólo que la filosofía gana en humildad, sino que a la vez el resto de las disciplinas conquistan su especificidad, su singularidad. ${ }^{7}$

¿Es sin embargo correcto afirmar que el concepto habla de sí mismo? Resignada su función representativa, parece imposible sostener que hable de cualquier cosa. ¿Y en qué medida podrá afirmarse que dos filósofos hablan de lo mismo? En efecto, abandonar la representatividad del concepto implica una redefinición de los objetivos de la filosofía. Por un lado, ya no se esperarán de ella explicaciones, sino acciones. En cuanto a la comunicación, será reemplazada por la creación. Las preguntas mismas cambiarán de valor: ¿cómo habrían de comunicar dos filósofos si son dos seres empeñados en crear conceptos? En otras palabras, ¿para qué hacer filosofía si podemos comunicar, es decir, si consideramos que un determinado andamiaje conceptual es suficiente y adecuado para resolver los problemas planteados? La filosofía, al contrario, comenzaría allí donde termina la comunicación, allí donde nuevos conceptos — nuevas herramientas - sean necesarios. En el fondo, una concepción performativista del concepto aspira a una mayor injerencia de la filosofía en el mundo, a una verdadera intervención, a una real inserción. Incluso habría que definir la explicación antes de resignarla y hacer de la representación su condición. En efecto, si admitimos que el concepto tiene una función constituyente de lo real, que su acción consiste, entre otras

${ }^{7}$ No por ello debemos deducir que las disciplinas se cierran sobre sí mismas y que se vuelve imposible sostener un discurso filosófico sobre el arte o la ciencia. Pero es cierto que deben reformularse tanto la epistemología como la estética dentro de los límites de la nueva metafilosofía. Deleuze y Guattari, por ejemplo, les asignarán la función de crear conceptos de afectos o conceptos de funciones respectivamente (Deleuze y Guattari, 1991, p. 188). 
cosas, en constituir la experiencia, al menos recibe ya una función epistémica. Es cierto que el mundo contemporáneo presenta formas, tal vez no más eficaces, pero más inmediatas y veloces de constituir de lo real. A nuestro juicio, esta es la primera dificultad que afronta una metafilosofía pragmatista. Desde el momento en que el concepto ya no habla por muchos y ni siquiera por algunos, desde el momento que no habla, sino que sólo actúa por sí, ¿cómo enfrentará el aluvión de imágenes y la tormenta de sonidos que confeccionan nuestra actualidad?, de manera microfísica o molecular, podríamos pensar con Guattari (1977: 14, 26 y 36). El concepto operará secretamente en nuestras maneras de pensar y su eficacia será la de una milicia incorpórea. Tal vez por eso devenga necesaria una alianza con otras fuerzas, una colaboración entre las disciplinas, los grupos, un esfuerzo colectivo para alcanzar una constitución multidimensional de lo real.

\section{Bibliografía}

Alliez, Éric, 1998, "Sur la philosophie de Gilles Deleuze: une entrée en matière", en Gilles Deleuze. Immanence et vie, París, PUF.

Bergson, Henri, 2003, La pensée et le mouvant, París, PUF. ,2003b, L'énergie spirituelle, París, PUF. ,2003c, L'évolution créatrice, París, PUF.

Deleuze, Gilles, 1968, Différence et répétition, París, PUF. , 1988, Le pli. Leibniz et le baroque, París, Minuit. , 2002, L'île déserte, París, Minuit. y Guattari, Félix, 1980, Mille plateaux. Capitalisme et Schizophrénie 2, París, Minuit. y Guattari, Félix, 1991, Qu’est ce que la philosophie?, París, Minuit. Descartes, René, 1979, Méditations métaphysiques, París, Flammarion. 
, 1996, Règles pur la direction de l'esprit, París, Vrin.

Foucault, Michel, 1994, “Theatrum philosophicum”, en Dits et écrits I, París, Gallimard.

Guattari, Félix, 1977, La révolution moléculaire, París, Recherches.

Guéroult, Martial, 1968, Spinoza II. L'àme, París, Aubier.

Jankélévitch, Vladimir, 1959, Henri Bergson, París, PUF.

Nancy, Jean-Luc., 1998, "Pli deleuzien de la pensée”, en Alliez, E., Gilles Deleuze. Une vie philosophique, París, Synthélabo (Les empêcheurs de penser en rond).

Platón, 1967, Cratyle, París, Flammarion.

, 1993, Le sophiste, París, Flammarion.

, 1965, Phédon, París, Flammarion.

Spinoza, Baruch, 1999, Éthique, París, Seuil. 